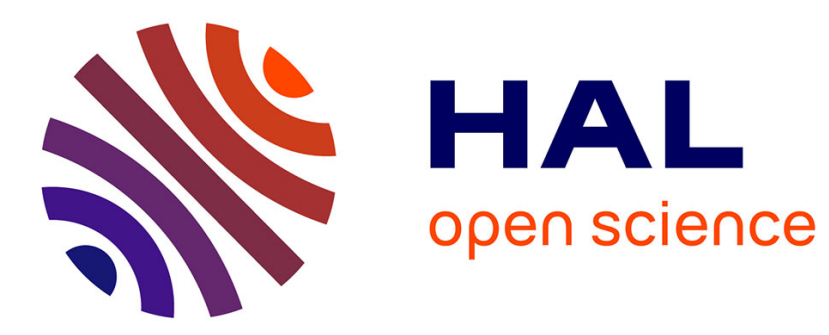

\title{
Collision analysis for an UAV
}

Anna Desilles, Hasnaa Zidani, Eva Crück

\section{To cite this version:}

Anna Desilles, Hasnaa Zidani, Eva Crück. Collision analysis for an UAV. AIAA GUIDANCE, NAVIGATION, AND CONTROL CONFERENCE, Aug 2012, Minneapolis, United States. pp.AIAA 2012$4526,10.2514 / 6.2012-4526$. hal-00756389

\section{HAL Id: hal-00756389 \\ https://hal.science/hal-00756389}

Submitted on 22 Nov 2012

HAL is a multi-disciplinary open access archive for the deposit and dissemination of scientific research documents, whether they are published or not. The documents may come from teaching and research institutions in France or abroad, or from public or private research centers.
L'archive ouverte pluridisciplinaire HAL, est destinée au dépôt et à la diffusion de documents scientifiques de niveau recherche, publiés ou non, émanant des établissements d'enseignement et de recherche français ou étrangers, des laboratoires publics ou privés. 


\title{
Collision analysis for an UAV
}

\author{
Anna Désilles, Hasnaa Zidani ${ }^{\dagger}$ \\ ENSTA ParisTech, Paris, 75015, France \\ Eva Crück ${ }^{\ddagger}$ \\ Délégation Générale pour l'Armement, 75509 Paris Cedex 15, France.
}

\begin{abstract}
The Sense and Avoid capacity of Unmanned Aerial Vehicles (UAV) is one of the key elements to open the access to airspace for UAVs. In order to replace a pilot's See and Avoid capacity such a system has to be certified" as safe as a human pilot on-board". The problem is to prove that an unmanned aircraft equipped with a S\&A system can comply with the actual air transportation regulations. This paper aims to provide mathematical and numerical tools to link together the safety objectives and sensors specifications.

Our approach starts with the natural idea of a specified "safety volume" around the aircraft: the safety objective is to guarantee that no other aircraft can penetrate this volume. We use a general reachability and viability concepts to define nested sets which are meaningful to allocate sensor performances and manoeuvring capabilities necessary to protect the safety volume. Using the general framework of HJB equations for the optimal control and differential games, we give a rigorous mathematical characterization of these sets. Our approach allows also to take into account some uncertainties in the measures of the parameters of the incoming traffic. We also provide numerical tools to compute the defined sets, so that the technical specifications of a S\&A system can be derived in accordance with a small set of intuitive parameters. We consider several dynamical models corresponding to the different choices of maneuvers (lateral, longitudinal and mixed). Our numerical simulations show clearly that the nature of used maneuvers is an important factor in the specifications of sensor's performances.
\end{abstract}

\section{Introduction}

Unmanned Aerial Vehicles (UAV) are widely used in current military operations. The development of the associated technologies has reached a point such that the question "Is it necessary to have a pilot on board?" can be asked for any airborne mission. However, there are several issues which need to be solved before UAVs can be allowed to fly routinely above our heads. The use of airspace is highly regulated, and the current regulatory structure has been inherited from a century of manned aviation. An evolution is necessary in order to accommodate unmanned aircraft without reducing the overall level of safety both on the ground and in the air. And such an evolution cannot be accepted if it imposes additional constraints onto traditional airspace users.

Among the key elements (see for instance ${ }^{1}$ ) to open the access to airspace for UAVs is the Sense and Avoid capacity (S\&A), which has to replace the human pilot See and Avoid capacity. Indeed, the absence of a qualified pilot on-board the aircraft shall not increase the probability of collisions for other airspace users. The design of a Sense and Avoid system likely to be certified "as safe as a human pilot on-board" is therefore a technological enabler for UAV operation outside segregated airspace. But the certification of such a system is an open regulatory issue. The current rather high level of safety of air transportation has been reached through intricate regulations under the assumption that a qualified pilot would be on-board

\footnotetext{
${ }^{*}$ Researcher, COMMANDS Team, ENSTA ParisTech \& Inria Saclay, UMA 32 Bd Victor, 75739 Paris Cx $15 . \quad$ Email: anna.desilles@ensta-paristech.fr

${ }^{\dagger}$ Professor, COMMANDS Team, ENSTA ParisTech \& Inria Saclay, UMA 32 Bd Victor, 75739 Paris Cx $15 . \quad$ Email: hasnaa.zidani@ensta-paristech.fr

${ }^{\ddagger}$ Research engineer, Navigation \& Guidance Department, eva.cruck@dga.defense.gouv.fr, Email: cruck@control.ee.ethz.ch, AIAA Member.
} 
each aircraft to control the flight. The problem is to prove that an unmanned aircraft equipped with a S\&A system can comply with these regulations. But what has turned out to be very difficult is to find acceptable ways for defining a safety target for a S\&A system, and for proving that this target has been reached. So the S\&A is not only a technological problem.

Several projects and working groups have provided elements for solving the S\&A issue. In the US, a workshop organized by $\mathrm{FAA}^{31}$ has established basic concepts but a lot more work is necessary to translate them into a technological standard and the associated means of compliance. An early attempt to set specifications for an S\&A system can be found $\mathrm{in}^{27}$ but without an associated safety target. A NATO working group, FINAS (flight in non-segregated airspace), has also drafted a stanag. It has not been possible to prove that compliance with these standards would be enough to gain access to unsegregated airspace. In Europe, the ongoing MIDCAS project ${ }^{2}$ is working toward a standard in close cooperation with the EUROCAE WG73 in an attempt to break this deadlock.

A lesson learnt from all the work so far is that a smooth way from safety objectives to sensors and algorithms, and back with means of compliances has to be defined in a way such that all the stake holders can understand it. In continuation of the work started in ${ }^{19}$, this paper aims to provide mathematical and numerical tools towards this objective. Our ambition is to bridge the gap between technical specifications for a S\&A system and a statement such as "The UAV shall not come close to another aircraft in a way likely to cause hazard.". From a global safety objective, it becomes possible to allocate target performances for a "sense" function and a "avoid" function in a tractable way.

Our approach starts with the natural idea of a specified "safety volume" around the aircraft: the safety objective is to guarantee that no other aircraft can penetrate this volume. Using tools from optimal control, viability theory and differential games, we define nested sets which are meaningful to allocate sensor performances and maneuvering capabilities necessary to protect the safety volume. We also provide numerical tools to compute these sets, so that the technical specifications of a S\&A system can be derived in accordance with a small set of intuitive parameters.

The mathematical formulation of the safety (or collision) regions is related to the reachable analysis of dynamical systems. This analysis seeks to partition the state space into two categories: those that are reachable from the initial conditions and those that are not. In the recent years, several contribution have been done dealing with the mathematical description of the reachable sets and their numerical computation. ${ }^{36,10,32,16,19,6}$ This growing interest stems from the importance for many applications to be able to accurately represent the reachable sets. The applications include navigation, aircraft conflict resolution, ${ }^{33,34,35,25,29}$ and vehicle collision avoidance. ${ }^{20}$

Several tools have been developed to describe the reachable sets. Among them the viability and HamiltonJacobi approaches provide a very general framework for non-linear systems without any controllability assumption. Moreover, these approaches can easily take into account state constraints and uncertainties, and provide efficient numerical methods, see, ${ }^{39,15,12,9,11}$ and the references therein, for more details.

This paper is organized as follows: The next section is devoted to the formal statement of the problem we need to solve. The mathematical model of this problem is derived in Section IV. The nested sets which are at the core of our approach are defined and characterized in Section III. A mathematical formulation of the safety set using the optimal control and viability setting is provided in Section V. The next two sections are devoted to numerical experiments in order to illustrate our approach. In Section VI, we study a simplified version of the problem for which all the parameters are known. Then in Section VII, we introduce uncertainties and we study how they modify the solutions to the simplified problem.

\section{Problem statement}

The "see and avoid" (S\&A) principle is embedded in the basis regulatory framework of airspace regulation. In Chapter 3 General rules of the Annex 2 Rules of the Air to the convention on international civil aviation, Section 3.2 Avoidance of collisions starts with the following:

Note. It is important that vigilance for the purpose of detecting potential collisions be not relaxed on board an aircraft in flight, regardless of the type of flight or the class of airspace in which the aircraft is operating, and while operating on the movement area of an aerodrome.

By law, the pilot in command of an aircraft is in charge of ensuring this vigilance; the "type of flight" and 
the "class of airspace" determine the conditions under which this responsibility is to be exerted and the support available from air traffic services.

It is impossible for an unmanned aircraft, whether or not a pilot is exerting control from the ground, to apply this principle in a strict sense. The expression "Sense and avoid" has been coined to denote a way, yet to be defined, to comply with Article 3.2.1 Proximity

An aircraft shall not be operated in such proximity to other aircraft as to create a collision hazard.

For about a decade, attempts have been made to translate this statement into technical specifications for equipment of UAVs. But as for now, no aviation authority has allowed an UAV to fly without special arrangements. Real-time imagery from cameras on-board has not been deemed sufficient. Generally, segregation in time or space is enforced so that no aircraft can be in the proximity of the UAV. Alternatives are ground-based surveillance systems covering the UAV flight range or a chase manned aircraft to ensure direct visual control of the airspace around the UAV.

In this paper, we are interested in specifying an airborne S\&A system. Starting from Article 3.2.1 above, we define conceptual and numerical tools to describe the "avoid problem" which are then linked to the "sense problem" in a tractable way.

\section{A. The "avoid" problem}

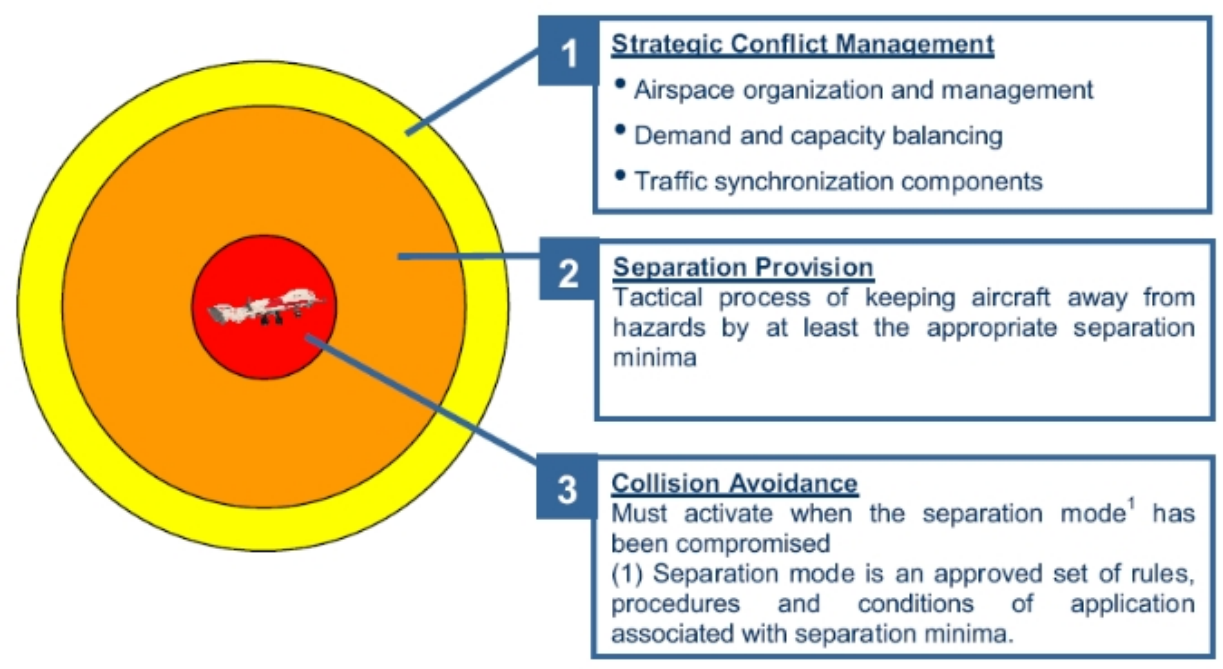

Figure 1. "See and Avoid" problem

The application of Article 3.2.1 to dense traffic has let to a layered approach which is notionally represented on Figure A. S\&A is concerned with the two inner layers. ${ }^{3,31,2}$ All the references agree on the fact that a S\&A system should provide two functions: a separation assurance function and a collision avoidance function. Separation assurance aims to stay "well clear" of other aircraft; collision avoidance is the emergency solution when separation assurance has failed. These general definitions raises two main questions:

- What does "well clear" mean in term of minimal distance between aircrafts?

- What are the conditions to declare that the collision avoidance is necessary?

It has to be noted that these notions have been inherited from the current operations of manned aviation. Separation assurance can under some conditions be provided by air traffic control (ATC) services, but collision avoidance is always the pilot's responsibility. Except for separation assurance provided by ATC, there exist no quantitative specification of the meaning of "well clear", and when a pilots maneuveres in order to avoid another aircraft, it is not always clear whether she is performing separation assurance or collision avoidance. 
The questions above have not received a definite answer for UAVs yet, but the different working groups have very similar approaches. The usual definition of a safe separation (if not provided by ATC) is the guarantee of a distance of the order of 0.5 nautical miles in the horizontal plane, and $500 \mathrm{ft}$ in the vertical dimension. ${ }^{3}$ Collision avoidance is generally associated with a miss distance of $500 \mathrm{ft}$ horizontally and $100 \mathrm{ft}$ vertically.

It follows that collision avoidance and separation assurance are both problems of keeping a prescribed volume around the UAV free of aircraft. So we can define the generic Avoid problem as keeping all traffic out of a volume denoted $\mathcal{O}$ centered on the $U A V$. In this paper, $\mathcal{O}$ is defined as a cylinder of radius $S e p_{h}$ and height $S e p_{v}$ but any 3 dimensional set can be used.

In order to be on the safe side, we shall assume that only the UAV will maneuver to avoid loss of separation or collision. This maneuver takes into account uncertainty on the trajectory of incoming traffic. The part of the Rules of the Air which determines which aircraft has the right of way are not taken into account here. We are dealing will the worst case situation when the pilot of the other aircraft is unaware of the UAV.

The range of available maneuvers depend not only on the UAV but also on the situation. Manoeuvring for separation assurance is a normal operation, so smooth maneuvers are required. In case of collision avoidance, sharper maneuvers are allowed since safety is at stake.

\section{B. The "sense problem"}

Because of the dynamics involved, incoming traffic has to be detected early enough. We are interested mostly in conflicts between fixed-wing aircraft, so the time between the initiation of an avoidance maneuver and its effect on the miss distance cannot be neglected.

Moreover, time has to be allowed for analyzing the situation to determine whether a maneuver is necessary. In addition, if a pilot has to decide a maneuver from a ground station, her reaction time and the transmission time have to be taken into account.

We assume that the relative dynamics of the aircraft can be measured or estimated. The precision of estimation is part of the parameters which can be specified using our approach.

In this paper, we are not interested in sensing technologies. Our purpose is to specify the sensing performances which are sufficient to solve the "avoid problem".

\section{Our approach for the S\&A problem}

Our aim is to be able to assess a given situation between two aircraft, given their positions and their dynamics and their possibilities of actions. We assume that only the trajectory of the UAV is controlled in order to avoid the collision. The incoming traffic is unaware of the UAV. For this purpose, we distinguish between three possible situations:

- $S_{1}$ : the loss of separation cannot be avoided,

- $S_{2}$ : the loss of separation can occur if no maneuver is initiated soon enough,

- $S_{3}$ : the separation is guaranteed without any maneuver.

These situations can be associated with regions in the state space of the problem. The dimension of the problem will be discussed when the dynamical models will be presented in the next section. In order to introduce the notions, we discuss here only the relative positions.

The interpretation of these sets is the following. The set corresponding to the first situation is the set to avoid absolutely. As it is shown on the figure 2(a) if an intruder is detected in this set (in red) then the loss of separation is unavoidable. If an intruder is detected in the complementary of this set, the collision can be avoided. It follows that the "avoid" problem can be solved only outside the set corresponding to the situation $S_{1}$. The consequence on the "sense problem" is that detection of aircraft close to the boundary of such a set must be certain. 
Now the "avoid" problem has been refined: it is necessary to be able to initiate a maneuver before entering the region of $S_{1}$. But an aircraft outside this region may not be on a conflicting trajectory. Therefore, it is important to determine the conditions under which a situation has to be considered as a conflict and a maneuver has to be initiated. For this purpose, we introduce a time parameter $t_{f}$ and we define a conflict as a situation in which an aircraft is likely to enter $S_{1}$ before time $t_{f}$. The associated set is shown on the figure $2(\mathrm{~b})$, in blue.

Whenever a traffic enters the set associated with the situation $S_{2}$, it becomes a potential threat, and it is necessary to be ready to initiate a maneuver. The earlier the detection, the smoother the maneuver to be taken. Therefore, the boundary of this set should be the minimum detection range for the "sense" problem.

\section{Mathematical characterization of different safety zones}

In order to give the precise mathematical characterization of all safety zones we distinguish two situations. In the first case, more simple, we assume that the parameters of the flight of the intruder are well known by the UAV. In the second case, more realistic, we assume that some parameters of the dynamics of the intruder are known with uncertainty. In each case we give a precise mathematical definition of the regions concerned.

Let us introduce some notations. Let $z(t) \in \mathbb{R}^{d}$ be the real vector that represents the state of an aircraft at any time $t>0$ : its position and its speed with respect to a given reference frame. Using some classical arguments of flight mechanics one can describe the evolution of an aircraft by a system of differential equations:

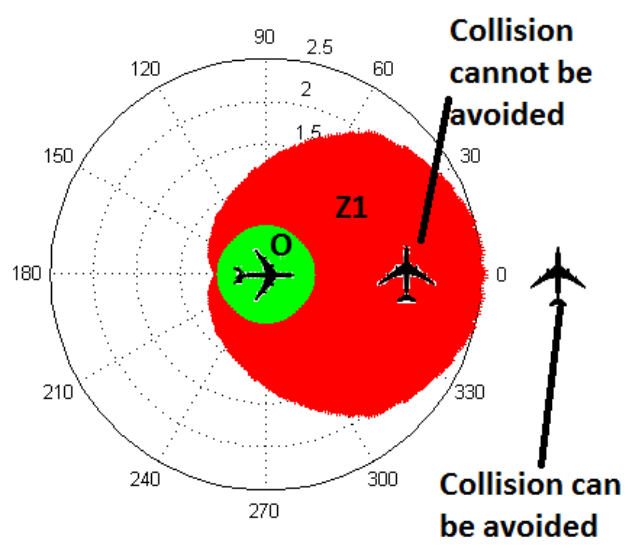

(a) Collision analysis with the set $Z_{1}$

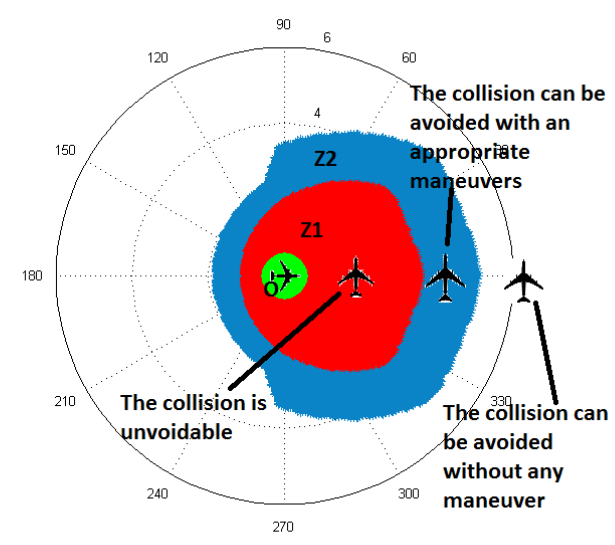

(b) Collision analysis with the set $Z_{2}\left(t_{f}\right)$

Figure 2. Collision analysis

$$
\begin{aligned}
& \dot{z}(s)=f(z(s), s, \alpha(s), \beta(s)), \text { for a.e. } s \in[0, t] \\
& z(0)=z_{0}
\end{aligned}
$$

with a given function $f$ corresponding to a chosen model of flight dynamics. It can depend on two vectors of controls of different kinds. The first one, $\alpha(\cdot)$ is the vector of UAV's control variables and the second, $\beta(\cdot)$ represents some uncertainties that one can take into account in the model.

Let $\mathcal{A}$ and $\mathcal{B}$ be two nonempty compact subsets of $\mathbb{R}^{m}$ and $\mathbb{R}^{p}$ respectively. Let $\mathbb{A}:=\{\alpha:(0, t) \rightarrow$ $\mathcal{A}$, measurable $\}$ and $\mathbb{B}:=\{\alpha:(0, t) \rightarrow \mathcal{B}$, measurable $\}$. For every $z_{0} \in \mathbb{R}^{d}$ and $(\alpha, \beta) \in \mathbb{A} \times \mathbb{B}$, we denote by $z=z_{z_{0}}^{\alpha, \beta}$ the associated trajectory defined as the (absolutely continuous) solution of (1).

Let $\mathcal{O} \subset \mathbb{R}^{d}$ be some open set that we will call "collision zone". All relative positions in this set are equivalent to collision. The precise definition of this set will depend from the choice of the state space for the dynamics. We will give it later. The collision zone $\mathcal{O}$ is considered here as an obstacle. Then its complementary set $\mathcal{K}=\mathbb{R}^{d} \backslash \mathcal{O}$ represents the set of state constraints. 


\section{A. Dynamics without uncertainties}

In the simplest case where all flight parameters of the UAV and of the intruder are assumed to be known precisely, the second control variable $\beta$ is constant and then

$$
f(z, s, \alpha, \beta)=f(z, s, \alpha)
$$

The trajectory of such a dynamical system, starting at a point $z_{0} \in \mathbb{R}^{d}$ with a control policy $\alpha(\cdot) \in \mathbb{A}$ will be denoted by $z_{z_{0}}^{\alpha}(\cdot)$.

Consider the situation where the UAV can do all the possible maneuvers to avoid the collision with infinite time horizon.

- The set $Z_{1}$ corresponding to the situation $S_{1}$ is defined as the subset of the state space where the collision is impossible to avoid. For any choice of control strategy the system reaches the collision zone.

$$
Z_{1}=\left\{z_{0} \in \mathcal{K} \mid \forall \alpha \in \mathbb{A} \quad \exists s \in \mathbb{R}^{+} \text {s.t. } z_{z_{0}}^{\alpha}(s) \in \mathcal{O}\right\}
$$

When the initial state of the system is in the set $\mathbb{R}^{3} \backslash Z_{1}$ we know that it is possible to maneuver to avoid collision. But an UAV has a specific mission and some nominal trajectory to follow with a nominal control $\alpha_{0}$.

Then it is interesting to determine the possibilities to avoid the collision without any maneuver.

- The set $Z_{2}\left(t_{f}\right)$, corresponding to the situation $S_{2}$, depends on a finite time horizon $t_{f}>0$ and is defined as the set of all initial states in $\mathbb{R}^{d} \backslash Z_{1}$ such that without any maneuver the system reaches the dangerous region $Z_{1}$ before $t_{f}$.

$$
Z_{2}\left(t_{f}\right)=\left\{z_{0} \in \mathcal{K}, \mid \exists s \in\left[0, t_{f}\right] \text { such that } z_{z_{0}}^{\alpha_{0}}(s) \in Z_{1}, \quad \text { and } \forall t \in[0, s], \quad z_{z_{0}}(t) \in \mathcal{K}\right\} .
$$

\section{B. Dynamics with uncertainties}

We can consider several kinds of uncertainties in this model, such as velocity estimate errors (intruder as well as UAV) or unexpected behavior of the intruder. The different security regions are defined as follows:

- The set $W_{1}$, corresponding to the situation $S_{1}$ is defined as the subset of the initial positions such that for any choice of control policy for the UAV it is not possible de guarantee the avoidance of the collision.

- The set $W_{2}\left(t_{f}\right)$, corresponding to the situation $S_{2}$ depends of a finite time horizon $t_{f}>0$ and is defined as the set of all initial states in $\mathbb{R}^{d} \backslash W_{1}$ such that without any maneuver there is a risk that the system reaches the region $W_{1}$ before $t_{f}$.

To give more precise definitions of these sets we put our problem into the game theoretic framework involving two players (player 1 being the UAV while the intruder plays the role of the second player), starting at time $t=0$. The set $W_{1}$ can be then described by the worst-case of a game when the first player (UAV) wants to avoid the collision zone $\mathcal{O}$, or equivalently to keep the system in the safe region $\mathcal{K}$ (set of constraints) using his input $\alpha$, while the second player tries to steer the system away from $\mathcal{K}$ (with his input $\beta)$.

As usual in the game theory, ${ }^{21}$ we define the set of non-anticipative strategies for the first player, as follows:

$$
\begin{gathered}
\Gamma:=\{a: \mathbb{B} \rightarrow \mathbb{A}, \forall(\beta, \tilde{\beta}) \in \mathbb{B} \text { and } \forall s \in[0, \infty], \quad(\beta(\theta)=\tilde{\beta}(\theta) \text { a.e. } \theta \in[0, s]) \Rightarrow \\
(a[\beta](\theta)=a[\tilde{\beta}](\theta) \text { a.e. } \theta \in[0, s]) \quad\} .
\end{gathered}
$$

In the sequel, we will restrict the choices of control laws for the UAV at the set $\Gamma$, which amounts saying that the strategies of this player are not influenced by the future choice of its adversary.

According to, ${ }^{14}$ we define here the victory domain for the first player (the UAV) as follows 
Definition III.1. We call victory domain for the first player the set $\mathcal{V}_{1}(\mathcal{K})$ of all initial positions $z_{0}$ such that exists a non anticipative strategy $a \in \Gamma$ such that for all admissible control $\beta \in \mathbb{B}$ the corresponding trajectory $z_{z_{0}}^{a[\beta], \beta}(t)$ avoids the collision set $\mathcal{O}$ for all $t \in[0,+\infty[$ :

$$
\mathcal{V}_{1}(\mathcal{K})=\left\{z_{0} \in \mathcal{K}, \mid \exists a \in \Gamma, \text { such that } \forall \beta(\cdot) \in \mathbb{B}, \quad \forall t \geq 0, z_{z_{0}}^{a[\beta], \beta}(t) \in \mathcal{K}\right\} .
$$

With this definition, we can now remark that

$$
W_{1}=\mathbb{R}^{3} \backslash \mathcal{V}_{1}(\mathcal{K})
$$

As in the case without uncertainties, the set $W_{2}\left(t_{f}\right)$ is defined for a system without maneuver, where the control variable $\alpha$ is fixed to a nominal control $\alpha_{0}$, so that

$$
f(z, s, \alpha, \beta)=f\left(z, s, \alpha_{0}, \beta\right)
$$

In this case the only control present in the equations is the uncertainty variable $\beta$. It follows that:

$$
W_{2}\left(t_{f}\right)=\left\{z_{0} \in \mathbb{R}^{d}, \quad \exists \beta(\cdot) \in \mathbb{B}, \exists s \in\left[0, t_{f}\right] \text { s.t. } z_{z_{0}}^{\alpha_{0}, \beta}(s) \in W_{1}\right\} .
$$

By definition the interpretation of the sets $W_{1}$ and $W_{2}\left(t_{f}\right)$ is different from the case without uncertainties. If an intruder is detected in the set $W_{1}$ the avoidance of the collision cannot be guaranteed. For all possible actions of the UAV there is a risk of collision, but it does'nt means that the collision will necessarily occur as when we consider the set $Z_{1}$. If an intruder is out of the set $W_{1}$ it is possible to find a strategy for the UAV that guarantee the avoidance of the collision. Also, if the intruder is in the set $W_{2}\left(t_{f}\right)$ for a given time horizon $t_{f}$ then there is a risk of collision if nothing is done to avoid it.

\section{Dynamics models for the collision avoidance problem}

\section{A. Point mass model for flight dynamics}

In this paper the Point Mass Model for general aircraft flight dynamic is used. Consider an inertial frame attached to the Earth. The state of the system is defined by the following six variables:

- $(x, y) \in \mathbb{R}^{2}$ is the horizontal position of the mass center of the aircraft in the inertial reference frame.

- $h \in \mathbb{R}_{+}$is the altitude

- $V \in\left[V_{\min }, V_{\max }\right]$ is the True Air Speed

- $\psi \in[-\pi, \pi]$ is the heading angle

- $\gamma \in[-\pi, \pi]$ is the flight pass angle (or velocity pitch)

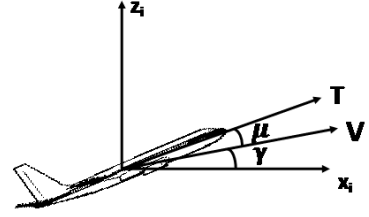

(a) Pitch attitude $\Theta=\mu+\gamma$

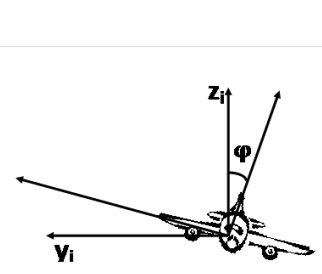

(b) Bank angle $\phi$

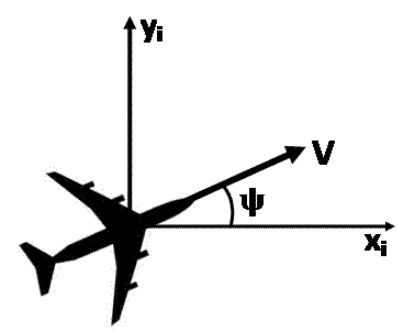

(c) Heading angle $\psi$

Figure 3. The angles used in the models 
The trajectory of the aircraft can be controlled by means of three controls : the bank angle $\phi \in\left[\phi_{\min }, \phi_{\max }\right]$, the thrust angle of attack $\mu \in\left[\mu_{\min }, \mu_{\max }\right]$, and the thrust $T \in\left[T_{\min }, T_{\max }\right]$. The definitions of the three angles used in the flight dynamics models are illustrated in the figure 3.

The problems of collision avoidance considered in this paper have generally very small time horizons. One can therefore assume that the fuel consumption is negligible and then that the mass $m$ of the aircraft is constant. The equations of the motion in the inertial reference frame are defined as follows:

$$
\begin{aligned}
& \dot{x}=V \cos (\gamma) \cos (\psi) \\
& \dot{y}=V \cos (\gamma) \sin (\psi) \\
& \dot{h}=V \sin (\gamma) \\
& \dot{V}=\frac{1}{m}(T \cos \mu-D-m g \sin \gamma) \\
& \dot{\psi}=\frac{1}{m V \cos \gamma}(T \sin \mu+L) \sin \phi \\
& \dot{\gamma}=\frac{1}{m V}((T \sin \mu+L) \cos \phi-m g \cos \gamma)
\end{aligned}
$$

where

$$
L=\frac{C_{L} S \rho(h) V^{2}}{2}, \quad D=\frac{C_{D} S \rho(h) V^{2}}{2}
$$

are respectively the lift and drag forces, $C_{D}$ and $C_{L}$ are respectively lift and drag coefficients, $S$ is the surface area of the wings and $\rho(h)$ is the density of the air, $g$ is the gravitational acceleration.

\section{B. Relative coordinates formulations for collision analysis}

Assume that before the detection of an intruder the UAV is in quasi-steady cruise flight. That means that its velocity $V_{u}$ is constant, the velocity vector is parallel to the ground $(\gamma=\mu=0)$. We are interesting here in the aircraft's motion during a short time interval when it can do some maneuvers to avoid a collision. For a short time horizon, the dynamics of the aircraft remains similar to a steady flight regime. Under this assumption some approximations can be considered in (7):

- $L \cos \left(\phi_{u}\right)=m_{u} g$

- $\mu_{u}^{2} \ll 1, \quad \cos \left(\mu_{u}\right) \simeq 1, \quad \sin \left(\mu_{u}\right) \simeq 0$

- $\gamma_{u}^{2} \ll 1, \quad \cos \left(\gamma_{u}\right) \simeq 1, \quad \sin \left(\gamma_{u}\right) \simeq 0$

- $T \mu \ll m g$

The details and the validity of these approximations can be found in. ${ }^{26}$

The velocity pitch angle $\gamma_{u}$ can be considered as a direct control instead of $\mu$. By neglecting the term $T \sin \mu$ in the equation for $\psi$ in (7) and tacking into account that $L=\frac{m g}{\cos (\phi)}$ and $\cos \left(\gamma_{u}\right) \simeq 1$ it yields:

$$
\dot{\psi}=\frac{g}{V_{u}} \tan (\phi)
$$

The angular velocity can be introduced by:

$$
\omega=\frac{g}{V_{u}} \tan (\phi)
$$

and considered as a new control variable instead of $\phi$. This new control takes its values in the interval $\omega \in\left[\omega_{\text {nim }}, \omega_{\max }\right]$ with

$$
\omega_{\min }=\frac{g}{V_{u}} \tan \left(\phi_{\min }\right), \quad \omega_{\max }=\frac{g}{V_{u}} \tan \left(\phi_{\max }\right) .
$$


With these approximations, the simplified dynamical system for the UAV in the inertial reference frame is:

$$
\begin{aligned}
\dot{x_{u}} & =V_{u} \cos \left(\gamma_{u}\right) \cos \left(\psi_{u}\right) \\
\dot{y_{u}} & =V_{u} \cos \left(\gamma_{u}\right) \sin \left(\psi_{u}\right) \\
\dot{h_{u}} & =V_{u} \sin \left(\gamma_{u}\right) \\
\dot{V_{u}} & =\frac{1}{m}\left(T-D-m g \sin \gamma_{u}\right) \\
\dot{\psi_{u}} & =\omega
\end{aligned}
$$

On the other hand, assume that the intruder aircraft is flying with a constant velocity $V_{a}$, constant heading angle $\psi_{a}$ and constant $\gamma_{a}=0$. His bank angle is then equal to zero: $\phi_{a}=0$. The equations of the motion of the intruder, with respect of the inertial reference frame are:

$$
\left\{\begin{array}{l}
\dot{x_{a}}=V_{a} \cos \left(\psi_{a}\right) \\
\dot{y_{a}}=V_{a} \sin \left(\psi_{a}\right) \\
\dot{h_{a}}=0
\end{array}\right.
$$

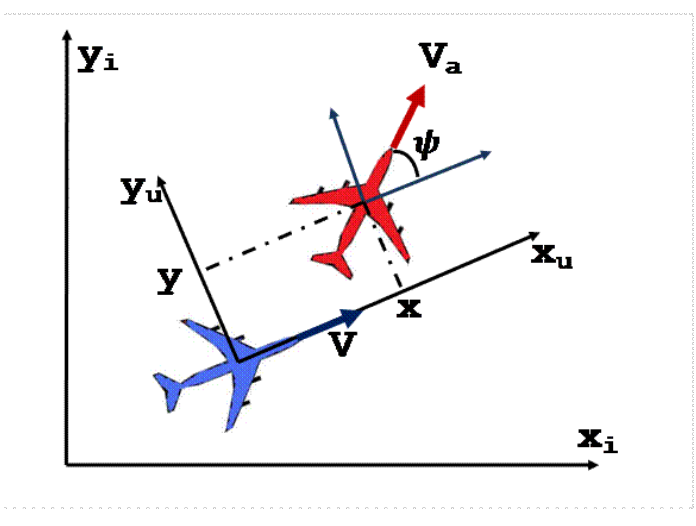

(a) $X Y$ projection

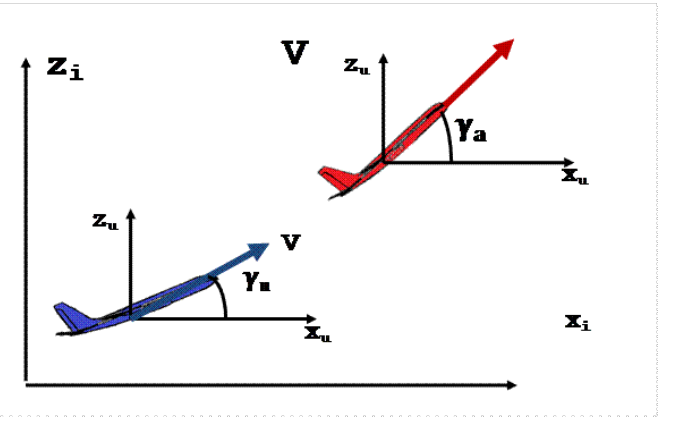

(b) $X Z$ projection

Figure 4. Definition of the local frame $\left(O_{u}, x_{u}, y_{u}, z_{u}\right)$ and of the relative position of the intruder:

From the perspective of the UAV the collision risk is evaluated based on the relative position of objects detected from the UAV. We will attach to the UAV a local reference frame defined as follows: it is obtained from the earth reference frame by translating its origin to the mass center of the UAV and by rotating it around of the $z$-axis by the angle $-\psi_{u}$ (the heading angle of the UAV). Then the $x$ axis of the new local frame coincides with the projection of the UAV's velocity vector into the $X Y$ plane.

Let $(x, y, h)$ be now the relative coordinates of the mass center of the intruder with respect to the local frame of the UAV. They a related to the coordinates in the inertial reference frame of two aircrafts as follows

$$
\left\{\begin{array}{l}
x=\left(x_{a}-x_{u}\right) \cos \psi_{u}+\left(y_{a}-y_{u}\right) \sin \psi_{u} \\
y=-\left(x_{a}-x_{u}\right) \sin \psi_{u}+\left(y_{a}-y_{u}\right) \cos \psi_{u} \\
h=h_{a}-h_{u}
\end{array}\right.
$$

and let $\psi=\psi_{a}-\psi_{u}$ be the relative heading. The figure 4(a) shows the $X Y$ projection of the new local frame. Its axis are named $\left(x_{u}, y_{u}, z_{u}\right)$ at the figure and the inertial reference frame axis are denoted $\left(x_{i}, y_{i}, z_{i}\right)$. It is shown here the relative horizontal position of the intruder $(x, y)$ and the relative heading angle $\psi$. The figure 4(b) shows the $X Z$ projection of the new local frame. It is shown here the relative vertical position of the intruder $h$ and the velocity pitch angles of both aircrafts $\gamma_{a}$ and $\gamma_{u}$. The dynamics equations in this 
new coordinate system become:

$$
\left\{\begin{array}{l}
\dot{x}=-V_{u} \cos \left(\gamma_{u}\right)+V_{a} \cos (\psi)+\omega y, \\
\dot{y}=+V_{a} \sin (\psi)-\omega x, \\
\dot{h}=-V_{u} \sin \left(\gamma_{u}\right), \\
\dot{V_{u}}=-\frac{C_{D} S \rho(h)}{2} \frac{V_{u}^{2}}{m_{u}}-g \sin \left(\gamma_{u}\right)+\frac{1}{m_{u}} T, \\
\dot{\psi}=\omega .
\end{array}\right.
$$

\section{Simplified models}

When an intruder is detected, the UAV can use three control variables to avoid collision:

- the angular velocity $\omega$ defines the horizontal maneuvers,

- the velocity pitch $\gamma_{u}$ defines the vertical maneuvers,

- and the trust $T$ defines the acceleration.

To study the importance of each of these control variables in the collision avoidance process we consider three simplified models.

- Model 1: Only vertical maneuvers are allowed. The heading angle $\psi=\psi_{0}$ is assumed to be constant. The control $T$ is used by the pilot to maintain the velocity constant. Then the aircraft's state is defined only by three variables: $(x, y, h)$ and the motion is described by the following system of equations.

$$
\left\{\begin{array}{l}
\dot{x}=-V_{u} \cos \left(\gamma_{u}\right)+V_{a} \cos \left(\psi_{0}\right) \\
\dot{y}=V_{a} \sin \left(\psi_{0}\right) \\
\dot{h}=-V_{u} \sin \left(\gamma_{u}\right)
\end{array}\right.
$$

The system is then controlled only by one variable, that is the velocity pitch $\gamma_{u}$.

- Model 2: Only horizontal maneuvers are allowed. One can assume that the aircraft is flying at a constant altitude $h$ and constant speed. Then $\gamma_{u}=0$ and In this context, the state of the system is reduced to the $3 \mathrm{D}$ vector $(x, y, \psi)$, and the equations of motion are

$$
\left\{\begin{array}{l}
\dot{x}=-V_{u}+V_{a} \cos (\psi)+\omega y \\
\dot{y}=V_{a} \sin (\psi)-\omega x \\
\dot{\psi}=-\omega
\end{array}\right.
$$

where the control variable is the angular velocity $\omega$.

- Model 3: Both vertical and horizontal maneuvers are allowed at constant speed. The control $T$ is used by the pilot to maintain the velocity constant. Then the aircraft's state is defined only by four variables: $(x, y, h, \psi)$ and the motion is described by the following system of equations. 
Here, the aircraft trajectory is controlled by the velocity pitch $\gamma_{u}$, and the angular velocity $\omega \in$ $\left[\omega_{\min }, \omega_{\max }\right]$.

$$
\left\{\begin{array}{l}
\dot{x}=-V_{u} \cos \left(\gamma_{u}\right)+V_{a} \cos (\psi)+\omega y \\
\dot{y}=V_{a} \sin (\psi)-\omega x \\
\dot{h}=-V_{u} \sin \left(\gamma_{u}\right) \\
\dot{\psi}=-\omega
\end{array}\right.
$$

where the system is controlled by two variables: $\omega$ and $\gamma$.

\section{Taking into account some uncertainties}

Some measures can be uncertain in our model. Likely the parameter that is most uncertain is the value of the intruder's speed. We will assume that it can be measured with an error $v \in\left[v_{\min }, v_{\max }\right]$ that will be considered as an additive uncertainty. The model equations are then modified as follows.

- Model 1. Here $\dot{\psi}=0, \dot{V}_{u}=0, \gamma_{a}=0$ then there are no angular velocity terms in the first two motion equations.

$$
\left\{\begin{array}{l}
\dot{x}=-V_{u} \cos \left(\gamma_{u}\right)+\left(V_{a}+v\right) \cos \left(\psi_{0}\right) \\
\dot{y}=\left(V_{a}+v\right) \sin \left(\psi_{0}\right) \\
\dot{h}=-V_{u} \sin \left(\gamma_{u}\right)
\end{array}\right.
$$

\section{- Model 2.}

$$
\left\{\begin{array}{l}
\dot{x}=-V_{u}+\left(V_{a}+v\right) \cos (\psi)+\omega y \\
\dot{y}=\left(V_{a}+v\right) \sin (\psi)-\omega x \\
\dot{\psi}=-\omega
\end{array}\right.
$$

- Model 3. Here $\dot{\psi}=0$ then there are no angular velocity terms in the first two motion equations.

$$
\left\{\begin{array}{l}
\dot{x}=-V_{u} \cos (\gamma)+\left(V_{a}+v\right) \cos (\psi)+\omega y \\
\dot{y}=\left(V_{a}+v\right) \sin (\psi)-\omega x \\
\dot{h}=-V_{u} \sin (\gamma) \\
\dot{\psi}=-\omega
\end{array}\right.
$$

\section{Optimal control theory: formulation of the S\&A problem}

In this paper, we propose to use the "level set" approach in order to characterize the safety sets $Z_{1}, Z_{2}$ or $W_{1}, W_{2}$. For each of these sets, we will consider a suitable control problem for which the value function can indicate for any position $z_{0}$ if it belongs to the safety set or not. The dynamical systems modeling the relative position of the intruder and the UAV are all of the form:

$$
\dot{y}=f(y(t), \alpha(t), \beta(t)),
$$

where $y$ denotes the state variable (which is of dimension 3 in the models 1 and 2 and in dimension 4 for the model 3). The control variable for the UAV is denoted $\alpha$, while $\beta$ the uncertainty of the model plays the 
role of the control for the second player. In the sequel, when the model is considered without uncertainties, $\beta$ will be set to 0 . In all our simplified models, the dynamics satisfies the following properties:

(H1) there exists $L_{f}>0$, such that for every $\left(x, x^{\prime}, a, b\right) \in \mathbb{R}^{d} \times \mathbb{R}^{d} \times \mathcal{A} \times \mathcal{B}$,

$$
\left|f(x, a, b)-f\left(x^{\prime}, a, b\right)\right| \leq L_{f}\left|x-x^{\prime}\right|,
$$

where $L_{f}>0$ is the Lipschitz constant.

(H2) For every $y \in \mathbb{R}^{d}$, and every $b \in \mathcal{A}, f(y, \mathcal{A}, b)$ is a convex set of $\mathbb{R}^{d}$.

\section{A. Problem without uncertainties}

In view of the properties of the dynamics, for any control variable $\alpha \in \mathcal{A}$ and for any initial condition $x \in \mathbb{R}^{d}$, there is a unique absolutely continuous trajectory $y_{x}^{\alpha}$ satisfying the system (17) with $y(0)=x$. For $t \in[0,+\infty]$, denote $S_{[0, t[}$ the set of all these trajectories starting at $x$ :

$$
S_{[0, t]}(x):=\left\{y:[0, t] \rightarrow \mathbb{R}^{d} \text { absolutely continuous, } y \text { satisfying (17) for some } \alpha \in \mathbb{A} \text {, and } y(0)=x\right\} .
$$

Moreover using the Ascoli-Alaoglu theorem, ${ }^{7}$ one can show that for any $t \in[0,+\infty], S_{[0, t]}$ is a compact set in $W^{1,1}\left(0, t ; e^{-\left(c_{0}+1\right) t}\right)$ (which is the space of functions $z \in L^{1}\left(0, t ; e^{-\left(L_{f}+1\right) t} d t\right)$ integrable for the measure $e^{-\left(L_{f}+1\right) t} d t$ and such that the weak derivative $\left.\dot{z} \in L^{1}\left(0, t ; e^{-\left(L_{f}+1\right) t} d t\right)\right)$ endowed with topology of $C([0, t])$.

\section{Characterization of $Z_{1}$}

Following some ideas introduced in, $, 4,11$ we consider a given parameter $\lambda>0$ and a Lipschitz continuous function $g: \mathbb{R}^{d} \rightarrow \mathbb{R}$ such that

$$
g(x) \leq 0 \quad \Leftrightarrow \quad x \in \mathcal{K} .
$$

Note that such a function always exists since we can choose $g(x):=d_{\mathcal{K}}(x)$, where $d_{\mathcal{K}}$ is the signed distance function $\left(d_{\mathcal{K}}(x)=-d(x, \partial \mathcal{K})\right.$ whenever $x \in \partial \mathcal{K}$ and $d_{\mathcal{K}}(x)=d(x, \partial \mathcal{K})$ if not). In the sequel, we choose $\lambda$ such that:

$$
\lambda>L_{f},
$$

where $L_{f}$ is the Lipschitz constant of $f$ (as given in H1). Now, consider the control problem:

$$
\vartheta(x):=\inf \left\{\max _{\theta \in[0,+\infty[}\left(e^{-\lambda \theta} g\left(y_{x}^{\alpha}(\theta)\right)\right), \alpha \in \mathbb{A}\right\} .
$$

Problem (19) is an infinite horizon control problem with supremum cost function. Here the term $\max _{\theta \in[0, \infty]} g\left(y_{x}^{\alpha}(\theta)\right)$ plays a role of a penalization that a trajectory $y_{x}^{\alpha}$ would pay if it violates the state-constraints. We will see in Theorem 2, that the advantage of considering (19) is that $\vartheta$ can be now characterized as the unique continuous solution of an HJB equation.

Theorem 1 (Characterization of the set $Z_{1}$ ). Let g be Lipschitz continuous function defined by (18). Let $\vartheta$ be the value function defined by (19). The the following statements hold:

(i) the unsafety set $Z_{1}$ is given by

$$
Z_{1}=\left\{x \in \mathbb{R}^{d}, \vartheta(x)>0\right\}
$$

(ii) if $\vartheta(x)<0$ and $\stackrel{\circ}{\mathcal{K}}=\{x, g(x)<0\}$, then there exists an admissible trajectory $y_{x}^{\alpha}$ which stays in the safe region $\mathcal{K}$ and never touches the boundary $\partial K$.

Proof. First, since the set of controls is compact and the function $g$ is Lipschitz, by Filippor theorem, for any $x \in \mathcal{K}$, the control problem admits an optimal solution. On the other hand, for $x$ in $\mathcal{K}$ such that $\vartheta(x) \leq 0$, it follows that:

$$
\begin{aligned}
\vartheta(x) \leq 0 & \Leftrightarrow \exists \bar{y}_{x} \in S_{[0,+\infty[}(x), \max _{\theta \in[0,+\infty[} e^{-\lambda \theta} g\left(y_{x}(\theta)\right) \leq 0, \\
& \Leftrightarrow \exists \bar{y}_{x} \in S_{[0,+\infty[}(x), y_{x}(\theta) \in \mathcal{K} \forall \theta \in[0,+\infty[, \\
& \Leftrightarrow x \notin Z_{1} .
\end{aligned}
$$


The proof of the statement (ii) is straightforward and can be obtained by the same arguments as for (i).

As mentioned before, the function $\vartheta$ can be characterized as the unique solution of a Hamilton-Jacobi equation. More precisely, considering the Hamiltonian

$$
H(x, p):=\max _{\alpha \in \mathcal{A}}(-f(x, \alpha) \cdot p)
$$

we have

Theorem 2. Under the property (H1), for any $\lambda>L_{f}$ and for any Lipschitz continuous $g$ satisfying (18), the value function $\vartheta$ is the unique continuous viscosity solution of the variational inequality (obstacle problem)

$$
\min (\lambda \vartheta+H(x, \nabla \vartheta), \vartheta-g(x))=0, \quad x \in \mathbb{R}^{d} .
$$

Proof The proof will be split into three parts.

Part 1: First, let us prove that the value function $\vartheta$ is Lipschitz continuous. For this, consider $x, x^{\prime} \in \mathbb{R}^{d}$. By using the simple inequalities:

$$
\max (A, B)-\max (C, D) \leq \max (A-C, B-D), \quad \text { and } \quad \inf (A, B)-\inf (C, D) \leq \sup (A-B, C-D),
$$

we get:

$$
\begin{aligned}
\vartheta(x)-\vartheta\left(x^{\prime}\right) & \leq \max _{\alpha \in \mathbb{A}} \max _{\theta \in[0,+\infty[} e^{-\lambda \theta}\left\|g\left(y_{x}(\theta)\right)-g\left(y_{x^{\prime}}(\theta)\right)\right\| \\
& \leq \max _{\alpha \in \mathbb{A}} \max _{\theta \in[0,+\infty[} e^{-\lambda \theta} L_{g} e^{L_{f} \theta}\left\|x-x^{\prime}\right\|
\end{aligned}
$$

where $L_{f}$ and $L_{g}$ are the Lipschitz constants respectively of $f$ and $g$. Therefore,

$$
\vartheta(x)-\vartheta\left(x^{\prime}\right) \leq \max _{\theta \in[0,+\infty[} e^{\left(L_{f}-\lambda\right) \theta} L_{g}\left\|x-x^{\prime}\right\|
$$

which with the condition $\lambda>L_{f}$ yields to

$$
\vartheta(x)-\vartheta\left(x^{\prime}\right) \leq L_{g}\left\|x-x^{\prime}\right\| \quad \forall x, x^{\prime} \in \mathbb{R}^{d} .
$$

This concludes the Lipschitz continuity of $\vartheta$.

Part 2: Dynamic programming principle (DPP). For definition of $\vartheta$, for any $x \in \mathbb{R}^{d}$ and $\tau>0$, the following holds:

$$
\vartheta(x):=\min _{\alpha=\left(\alpha_{1}, \alpha_{2}\right) \in \mathbb{A}}\left(\left(\max _{\theta \in[0, \tau[} e^{-\lambda \theta} g\left(y_{1}^{\alpha}(\theta)\right)\right) \bigvee \max _{\theta \in[\tau,+\infty[} e^{-\lambda \theta} g\left(y^{\alpha}(\theta)\right)\right)
$$

By classical arguments, we conclude that:

$$
\vartheta(x):=\min _{\alpha \in \mathbb{A}}\left(\left(\max _{\theta \in[0, \tau[} e^{-\lambda \theta} g\left(y_{1}^{\alpha}(\theta)\right)\right) \bigvee e^{-\lambda \tau} \vartheta\left(y^{\alpha}(\theta)\right), \forall \tau \geq 0\right.
$$

Part 3: We now check that $\vartheta$ is a solution of the HJB equation. By the DPP (see Part 2), we get that for any $\tau \geq 0$

$$
\vartheta(x) \geq \inf _{\alpha \in \mathbb{A}} e^{-\lambda \tau} \vartheta\left(y_{x}^{\alpha}(\tau)\right) .
$$

Hence, with classical arguments, we can obtain

$$
\lambda \vartheta+H(x, \nabla \vartheta) \geq 0
$$

in the viscosity sense. Moreover, by definition of $\vartheta$, for every $x \in \mathbb{R}^{d}$, we have

$$
\vartheta(x) \geq \inf _{\alpha \in \mathbb{A}} \max _{\theta \in[0, \infty]} e^{-\lambda \theta} g\left(y_{x}^{\alpha}(\theta)\right) \geq g(x) .
$$


Combining these two inequalities, we get

$$
\min (\lambda \vartheta+H(x, \nabla \vartheta), \vartheta(x)-g(x)) \geq 0
$$

in the viscosity sense, i.e., $\vartheta$ is a supersolution of $(22)$.

Let us now prove that $\vartheta$ is a subsolution. Let $x \in \mathbb{R}^{d}$. If $\vartheta(x)=g(x)$, it is obvious that $\vartheta$ satisfies:

$$
\min (\lambda \vartheta+H(x, \nabla \vartheta), \vartheta(x)-g(x)) \leq 0 .
$$

Now, assume that $\vartheta(x)>g(x)$. By continuity of $g$ and $\vartheta$, there exists some $\tau>0$ such that $e^{-\lambda \tau} \vartheta\left(y_{x}^{\alpha}(\theta)\right)>$ $e^{-\lambda \theta} g\left(y_{x}^{\alpha}(\theta)\right.$ ) for all $\theta \in[0, \tau]$ (since $y_{x}^{\alpha}(\theta)$ will stay in a neighborhood of $x$ which is controlled uniformly with respect to $\alpha$ ). Hence, by using the DPP, we get that

$$
\vartheta(x)=\inf _{\alpha \in \mathbb{A}} e^{-\lambda h} \vartheta\left(y_{x}^{\alpha}(h)\right), \quad \text { for any } 0 \leq h \leq \tau .
$$

We then deduce by classical arguments ${ }^{8}$ that $\lambda \vartheta(x)+H(x, \nabla \vartheta(x, t)) \leq 0$ in the viscosity sense. Therefore, $\vartheta$ is a viscosity subsolution of $(22)$.

The fact that $\vartheta$ is the unique solution of the equation (22) follows from the comparison principle for the equation (22) (see for instance ${ }^{28,4}$ ), and the fact that the Hamiltonian function $H$ satisfies

$$
\begin{gathered}
\left|H\left(x_{2}, p\right)-H\left(x_{1}, p\right)\right| \leq C(1+|p|)\left|x_{2}-x_{1}\right|, \\
\left|H\left(x, p_{2}\right)-H\left(x, p_{1}\right)\right| \leq C\left|p_{2}-p_{1}\right|,
\end{gathered}
$$

for some constant $C \geq 0$ and for all $x_{i}, p_{i}, x$ and $p$ in $\mathbb{R}^{d}$.

Remark 1. Inequalities such as (22) appear also in the framework of exit time problems where the obstacle $g$ represent the exit cost that should be paid for exit. Here, $g$ is a "fictitious cost" that a trajectory would pay if it leaves $\mathcal{K}$.

It is worth mentioning that theorem 2 is valid for any choice of a Lipschitz function $g$ satisfying (18) and for any $\lambda>L_{f}$. Of course, the value function $\vartheta$ is dependent on $g$ and $\lambda$, while the set $\left\{x \in \mathbb{R}^{d}, \vartheta(x) \leq 0\right\}$ does not depend neither on $g$ not on $\lambda$.

In view of theorem 2 , the set $Z_{1}$ can be fully described if we are able to compute the value function $\vartheta$. And indeed, $\vartheta$ is the unique solution of a HJB equation and it can be obtained by numerical approximation of (22). A huge literature is devoted to the numerical schemes for the discretionary of HJB equations. ${ }^{13,12,17,38,23,22}$ Here in our computation experiments, we shall use a classical scheme based on fixed point algorithm and ENO scheme. More precisely, we shall approximate $\vartheta$ by the following algorithm (where $h$ plays a fictitious time step and $\mathcal{G}$ is a given grid on $\mathbb{R}^{d}$ ):

Algorithm 1 for $Z_{1}$ :

- For $k=0$, consider $v^{0}$ a given function on the domain of computation $\mathcal{G}$

- For $k \geq 1$, compute $v^{k}$ by:

$$
v^{k}(x)=\max \left((1-\lambda h) v^{k-1}-\mathcal{H}^{\operatorname{num}}\left(x, D^{+} v^{k}(x), D^{-} v^{k}(x)\right), g(x)\right) \quad \text { for } x \in \mathcal{G},
$$

where $\mathcal{H}^{\text {num }}(x, p, q)$ is a numerical Hamiltonian which approximate the true Hamiltonian $H$, and $D^{+} v^{k}(x), D^{-} v^{k}(x)$ are respectively the finite differences on the grid $G$ decentralized respectively to the right and to the left. ${ }^{5,11}$

The set $Z_{1}$ can be computed also using the viability algorithms. Indeed, the definition of this set given in (3) can be rewritten as follows

$$
Z_{1}=\mathbb{R}^{d} \backslash\left\{z_{0} \in \mathcal{K} \mid \exists \alpha \in \mathbb{A} \quad \forall s \in \mathbb{R}^{+} \quad z_{z_{0}}^{\alpha}(s) \in \mathcal{K}\right\}=\mathbb{R}^{d} \backslash \operatorname{Viab}(\mathcal{K})
$$

This defines the set $Z_{1}$ as a complementary set of the viability kernel, mboxViab(K), of the constraints set $\mathcal{K}=\mathbb{R}^{d} \backslash \mathcal{O}$ (see for example ${ }^{6}$ for the definitions of the viability theory). One can compute the viability kernel using the set-valued algorithms defined in ${ }^{40}$ and take it's complementary set as an approximation of the set $Z_{1}$. In the case of the dynamics without uncertainties the differential equation (1) can be written as a differential inclusion of the form

$$
z^{\prime}(t) \in F(z(t)), \quad x \in \mathcal{K}
$$


with a set-valued function $F$ defined by

$$
F(z)=\{f(z, \alpha), \quad \alpha \in \mathcal{A}\}
$$

Let $\rho>0$ be a discretization step with respect to the time variable $t$. We associate with the differential inclusion (26) the explicit Euler scheme:

$$
\frac{x_{n+1}-x_{n}}{\rho} \in F\left(x_{n}\right), \quad x_{n} \in \mathcal{K}
$$

Let $G_{\rho}$ be the application defined as follows:

$$
G_{\rho}(x)=x+\rho F(x)+\varepsilon(\rho) B
$$

where $B$ is a unit ball in $\mathbb{R}^{d}$ and $\varepsilon(\rho)$ is a constant that is chosen to assure the convergence of the algorithm. The viability kernel algorithm is defined as follows:

Algorithm 2 for $Z_{1}$ :

- For $k=0, K_{0}=\mathcal{K}$

- For $k \geq 1$, compute:

$$
K_{n+1}=G_{\rho}^{-1}\left(K_{n}\right) \cap K=\left\{x \in K_{n}, \text { t.q } G_{\rho}(x) \in K_{n}\right\}
$$

\section{Characterization of $Z_{2}\left(t_{f}\right)$}

For $t_{f}>0$, the set $Z_{2}\left(t_{f}\right)$ corresponds to all the initial positions from which the system reaches the dangerous region $Z_{1}$ if no maneuver is undertaken (see the definition 4). Here the control variable is $\alpha \equiv 0$, and the dynamics of the systems is $f(x, 0,0)=: f_{0}(x)$. For each initial position there is only one trajectory that will be denote $y_{x}^{f_{0}}$ satisfying

$$
\dot{y}(t)=f_{0}(y(t)), \quad y(0)=x .
$$

Then the set $Z_{2}\left(t_{f}\right)$ is known also as the capture basin of $Z_{1}$ under the dynamics $f_{0}$. This capture basin can also be computed by a level-set approach, see. ${ }^{37,11}$

\section{B. The game theoretical approach for dynamics with uncertainties}

\section{Characterization of $W_{1}$}

As for the characterization of the set $Z_{1}$, it is also possible to construct a control problem for which the value function allows to characterize the set $W_{1}$. The later consists of all initial positions from which for any control policy for the UAV, there exists a risk of collision. This risk comes from the fact that the model of the intruder contains some uncertainties. So the set of $W_{1}$ is the set of the "worst cases". It can be represented by the value function of a two-player game problem, where the player 1 (UAV) uses his control policies and the second player is the intruder using the uncertainties as inputs. As mentioned in section B, the admissible strategies for the UAV are non-anticipative.

As in (19), we consider any Lipschitz continuous function $g$ satisfying (18) and any $\lambda>L_{f}$. The control problem is:

$$
\vartheta^{\text {unc }}(x):=\min _{a \in \Gamma} \max _{\beta \in \mathbb{B}}\left\{\max _{\theta \in[0,+\infty[}\left(e^{-\lambda \theta} g\left(y_{x}^{a[\beta], \beta}(\theta)\right)\right)\right\} .
$$

This setting includes of course the one-player game (it suffices to take $B=\{0\}$, then any $\alpha \in \mathbb{A}$ represents an admissible non-anticipative strategy).

Theorem 3 (Characterization of $\left.W_{1}\right)$. Under the properties (H1)-(H2). Let $\vartheta$ unc be the value function defined by (29). We have:

$$
W_{1}=\left\{x \in \mathbb{R}^{d}, \vartheta^{u n c}(x)>0\right\} .
$$

Moreover $\vartheta^{u n c}$ is the unique continuous viscosity solution of the Hamilton-Isaac equation:

$$
\min \left(\lambda \vartheta^{u n c}+H^{u n c}\left(x, \nabla \vartheta^{u n c}(x)\right), \vartheta^{u n c}(x)-g(x)\right)=0 \quad \text { for } x \in \mathbb{R}^{d},
$$

where $H^{\text {unc }}(x, p)=\max _{a \in \mathcal{A}} \min _{b \in \mathcal{B}}(-f(x, a, b) \cdot p)$. 
The proof of this theorem fellows the same arguments as for (2). The consequence of this result is that an approximation of set $W_{1}$ can be obtained by computing an approximation of the value function $\vartheta^{\text {unc }}$. For this, a discrete approximation of (30) can be done by using efficient numerical algorithms known in the literature. ${ }^{13,18,24,30}$

The viability procedure to compute the set $W_{1}$ is similar to the algorithm for the set $Z_{1}$. We define the set-valued map

$$
F(x, \beta)=\left\{\begin{array}{l}
\bigcup_{\alpha \in \mathbb{A}} f(x, \alpha, \beta), \text { if } x \in \mathcal{K} \\
\overline{C o}\left[\{0\} \cup \bigcup_{\alpha \in \mathbb{A}} f(x, \alpha, \beta)\right], \text { otherwise }
\end{array}\right.
$$

and it's Euler approximation (for some time step $\rho$ :

$$
G_{\rho}(x, \beta)=x+\rho F(x, \beta)+\varepsilon(\rho) B
$$

where $B$ is a unit ball in $\mathbb{R}^{d}$ and $\varepsilon(\rho)$ is a constant that is chosen to assure the convergence of the algorithm. The algorithm to compute the set $W_{1}$ is defined as follows:

Algorithm 3 for $W_{1}$ :

- For $k=0, K_{0}=\mathcal{K}$

- For $k \geq 1$, compute:

$$
K_{n+1}=\left\{x \in K_{n} \mid \forall \beta \in \mathbb{B}\left(G_{\rho}(x, \beta) \cap K_{n}\right) \neq \emptyset\right\}
$$

\section{Characterization of $W_{2}\left(t_{f}\right)$}

For $t_{f}>0$, the set $W_{2}\left(t_{f}\right)$ corresponds to all the initial positions from which the system reaches the region $W_{1}$ if no maneuver is undertaken (see the definition 6 ). Here the control variable is $\alpha \equiv 0$, and the dynamics of the systems is $f(x, 0, \beta)=: f_{1}(x, \beta)$. The system is controlled only by the uncertainty variable $\beta \in \mathbb{B}$. Then the set $W_{2}\left(t_{f}\right)$ can be described as the capture basin under the dynamics $f_{1}$ with the target set $W_{1}$. This capture basin can also be computed by a level-set approach, see ${ }^{37,11}$ or a corresponding viability algorithm, see. $^{40}$

\section{Numerical simulations for the case without uncertainties}

To compute and analyze all the defined sets corresponding to different levels of security we use two numerical approaches. The first one is based on the viability theory $\left(\mathrm{see}^{39}\right)$. The second approach is based on the numerical resolution of the HJB equations corresponding to the problem as described in previous sections.

\section{A. Data for the case study}

In all numerical simulations that are presented here we consider a MALE type UAV with following aerodynamic characteristics:

Table 1. Flight dynamics parameters

\begin{tabular}{|c|c|c|c|c|c|c|c|c|c|c|}
\hline Parameter & $V$ & $\phi_{\max }$ & $\mathrm{S}$ & $\mathrm{m}$ & $C_{L}$ & $C_{D}$ & $h$ & $T_{\text {nom }}$ & $\omega_{\max }$ & $\gamma_{\max }$ \\
\hline Value & 150 & 18 & 15 & 4000 & 0.3 & 0.029 & 5 & 4000 & 2.4 & 5 \\
\hline Units & $\mathrm{nm} / \mathrm{h}$ & & $m^{2}$ & $\mathrm{~kg}$ & & & $\mathrm{~nm}$ & $\mathrm{~N}$ & $\mathrm{rad} / \mathrm{min}$ & \\
\hline
\end{tabular}

where $\phi_{\max }$ is the maximum bank angle and $T_{\text {nom }}$ is the nominal thrust. For the numerical simulations we take for time unit one minute and for distance unit a nautical mile (nm). The maximum angular velocity 
is derived from the bank angle as follows:

$$
\omega_{\max }=\frac{g}{V} \tan \phi_{\max }
$$

The parameters defining the computational domains for the different models are given in the following table:

Table 2. Flight dynamics parameters

\begin{tabular}{|c|c|c|c|c|c|c|c|}
\hline Parameter & $x_{\min }$ & $x_{\max }$ & $y_{\min }$ & $y_{\max }$ & $R$ & $h_{\text {sep }}$ & $t_{f}$ \\
\hline Value & -6 & 6 & -6 & 6 & 0.5 & 0.1 & 0.25 \\
\hline Units & $\mathrm{nm}$ & $\mathrm{nm}$ & $\mathrm{nm}$ & $\mathrm{nm}$ & $\mathrm{nm}$ & $\mathrm{nm}$ & $\min$ \\
\hline
\end{tabular}

All the computations was performed on a Intel Core i5 CPU, $2.8 \mathrm{GHz}$, under Windows or Linux 64bit operating systems. For the models without uncertainties we show the results obtained with the viability algorithms. And the numerical study of the uncertain model is illustrated with the results from HJB codes.

\section{B. Lateral avoidance}

In all considered examples the speed of the UAV is fixed to $V_{u}=150$ knots. The dynamics used here is defined in (12). The system is controlled by $\omega$, the angular speed of the UAV. The control set $\mathbb{A}=[-2.4,2.4]$. The collision zone for this model is the cylinder $\mathcal{O}=B_{2}(R) \times[0,2 \pi]$ where $B_{2}(R)$ is the disc in the plane $X Y$ of radius $R$ centered at the origin.
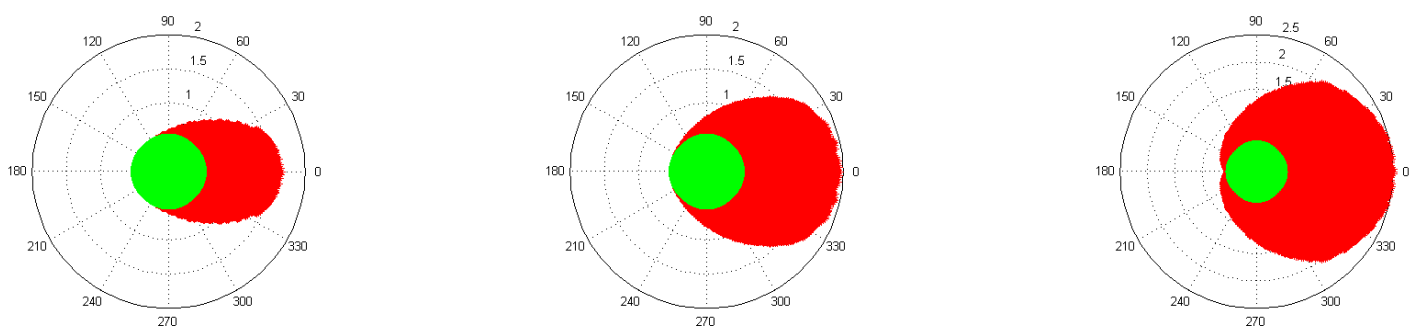

(a) $V_{a}=90$ knots, projection in $X Y$ plan (b) $V_{a}=150$ knots, projection in $X Y$ plan (c) $V_{a}=210$ knots, projection in $X Y$ plan

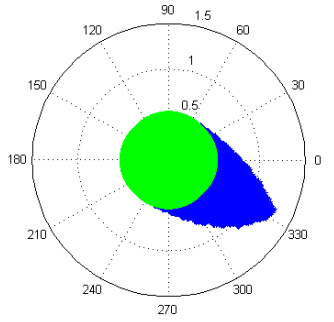

(d) $V_{a}=90$ knots, section with $\psi=\pi / 2$
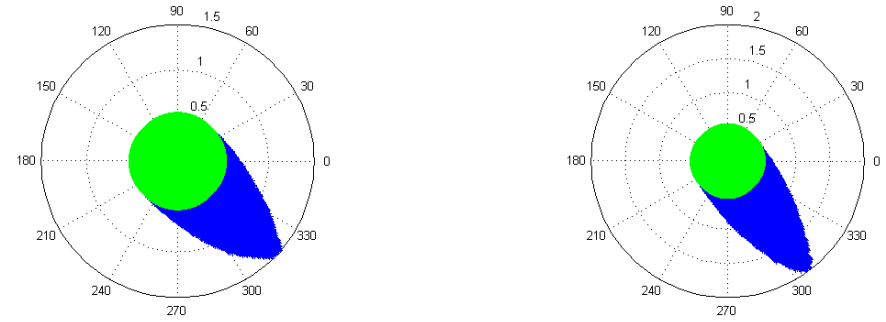

Figure 5. $Z_{1}$ sets for different values of the intruder's speed

The figure 5 presents the representations of the unsafety region $Z_{1}$ in the flight plan $(x, y)$ for different values of the intruder's speed $V_{a}$. The collision zone $\mathcal{O}$ is in green. The subfigures (a), (b) and (c) represent the projections of the sets $Z_{1}$ into the flight plan. Such a projection is particularly important to calibrate the sensors. It contains all the initial relative horizontal positions of the intruder for which the collision cannot be avoided, for all possible relative heading angle. The range of detection of the sensors may be determined 
using these projections. In a real situation, when the relative heading is known the corresponding set $Z_{1}$ can be refined as it is shown on the subfigures (d), (e) and (f).

The study of different values of the intruder's speed is also important here. The examples shown on the figure 5 on the previous page confirm the sensitivity of the region $Z_{1}$ with respect to the relations between the speeds of two aircrafts. When the intruder is faster then the UAV ( see (c)) the region $Z_{1}$ is the largest one. When defining the range of the sensors of the UAV it is necessary to take into account different classes of aircrafts that it can meet during its mission.

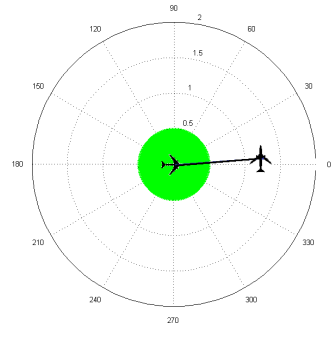

(a) Detection of an intruder

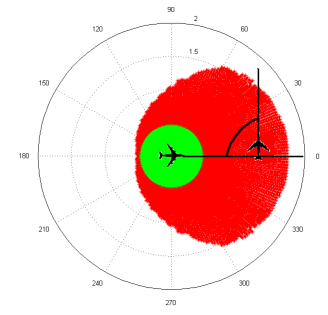

(b) Estimation of the relative heading

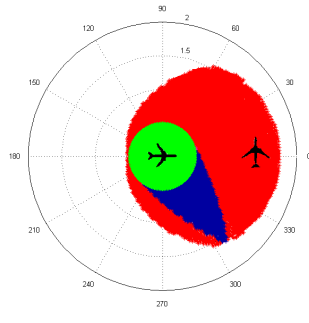

(c) The collision can be avoided

Figure 6. Analysis of a conflict situation

When an aircraft is detected by the UAV the first two values that are used to appreciate the importance of the danger are the distance and the speed of the intruder (see figure 6(a)). The $X Y$ projection of set $Z_{1}$ corresponding to the measured speed value $V_{a}$ can be used to determine if the collision can be avoided. If the intruder is in this set (see figure 6(b)) then the relative heading is used to make a more precise analysis. In the example shown on the figure $6(\mathrm{c})$ the relative heading is $\psi=\pi / 2$ and the intruder is out of the corresponding region $Z_{1}$ ( in blue). In this case the collision can be avoided.

When the intruder's position is out of the set $Z_{1}$ one can refine the analysis using the set $Z_{2}(T)$. The figure 7 shows an example of the set $Z_{2}(T)$ for $V_{a}=V=210$ knots and $T=0.25 \mathrm{~min}$.

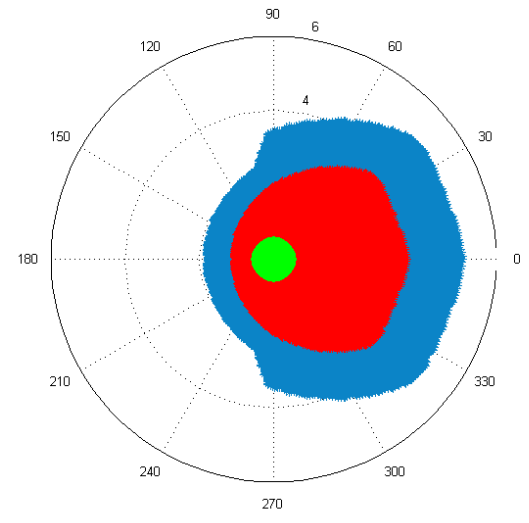

(a) Projection in $X Y$ plan: $Z_{1}$ in red, $Z_{2}(0.25)$ in yellow

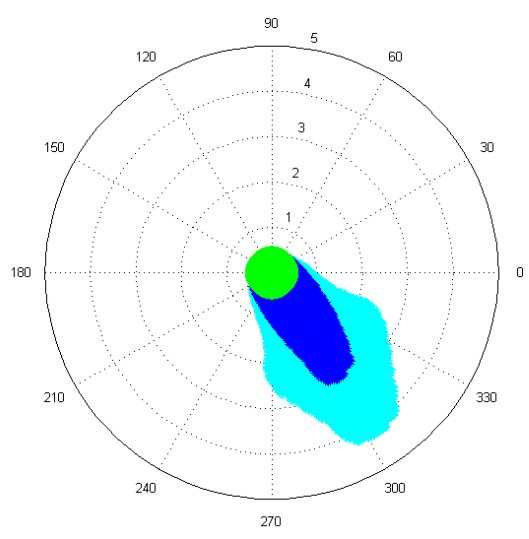

(b) Section with $\psi=\pi / 2: Z_{1}$ in blue, $Z_{2}(0.25)$ in cyan

Figure 7. The set $Z_{2}(T)$ with $T=0.25$ and $V_{a}=3.5$

The figure 8 on the following page illustrates the interpretation of the set $Z_{2}(T)$ in different situations. On the subfigure (a) the relative heading is $\psi=\pi / 2$ and the intruder is out of the region $Z_{2}(T)$ for $T=0.25$ min. That means that the collision can be avoided without any maneuver before the time $T$. At the figure (b) the intruder is in the set $Z_{2}(T)$. In this situation it is possible to avoid the collision but the UAV must 
engage avoidance maneuvers to do it. At the figure (c) the intruder is out of the projection of the set $Z_{2}(T)$ into the flight plan. That means that for any relative heading the collision can be avoided without any maneuver.

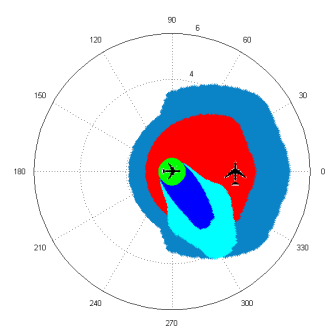

(a) Collision can be avoided without maneuver if $\psi=\pi / 2$

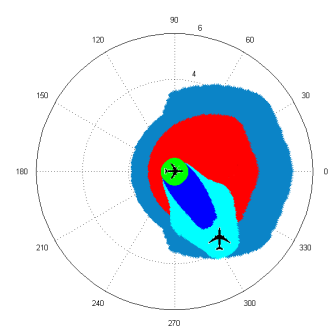

(b) Collision can be avoided with some maneuver if $\psi=\pi / 2$

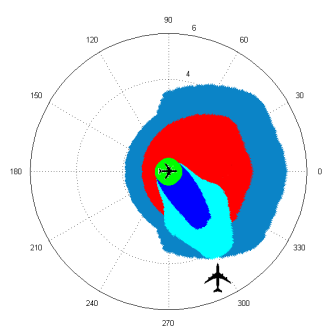

(c) Collision can be avoided without maneuver for any $\psi$

Figure 8. Collision analysis with different relative positions of the intruder

\section{Other models for the avoidance maneuvers}

One can do the same detailed analysis of the sets $Z_{1}$ and $Z_{2}$ with other models for the dynamics of the aircrafts. In this section we focus on the comparison of the results obtained with different models. Let us consider the case when the intruder has the same speed that the UAV: $V_{a}=V=150$ knots. The figure 9

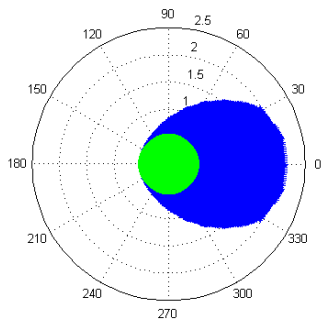

(a) Lateral avoidance, projection in $X Y$ plan

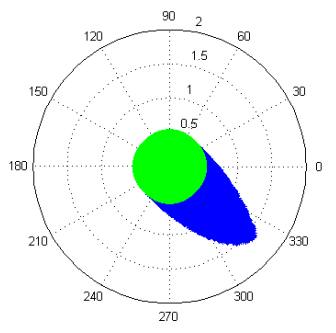

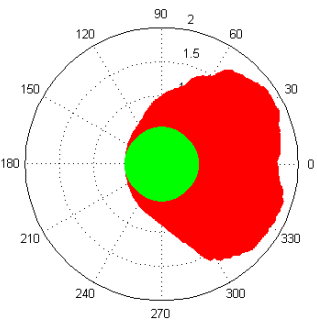

plan

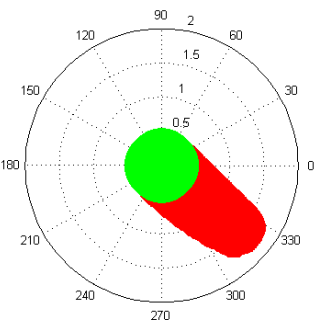

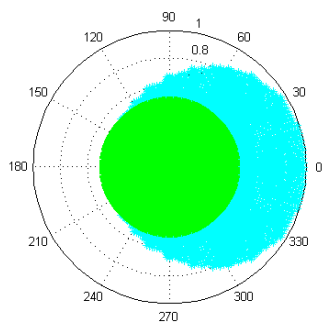

(c) 4D model, projection in $X Y$ plan

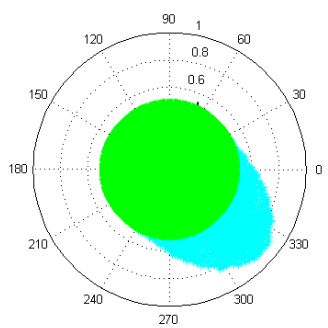

(d) Lateral avoidance, section with $\psi=$ (e) Vertical avoidance, section with $\psi=$ (f) $4 \mathrm{D}$ avoidance model, section with $\psi=$ $\pi / 2$ $\pi / 2$ $\pi / 2 \mathrm{n}$

Figure 9. $Z_{1}$ sets for different dynamics models

shows the sets $Z_{1}$ calculated with different assumptions about the choice of maneuvers. The figures (a), (b), (c) present the projections of the corresponding sets for the models 1,2 and 3 respectively into the plane 
$X Y$ (all values of the relative heading $\psi$ and all values of the relative altitude $h$ for the models with vertical avoidance). The figures (d), (e), (f) show the corresponding set (for the models 1,2 and 3 respectively ) with the relative heading fixed to $\psi=\pi / 2$.

One can conclude here that the choice of the allowed maneuvers is an important factor in the definition of the unsafety sets around the UAV and then in the definition of the range of detection of the sensors. The smallest regions $Z_{1}$ correspond to the case when both vertical and lateral avoidance maneuvers can be used. This result can also allow to refine the conflict analysis by taking into consideration an additional maneuver.

The figure 10 shows the sets $Z_{1}^{\text {lateral }}$ (in blue), $Z_{1}^{\text {vertical }}$ ( in red) and $Z_{1}^{\text {both }}$ (in cyan). When an intruder is detected in the set $Z_{1}^{\text {lateral }}$ it is impossible to avoid the collision by using only the lateral maneuvers. But if the intruder is in the set $Z_{1}^{\text {lateral }} \backslash Z_{1}^{\text {both }}$ it is steel possible to avoid the collision by using more maneuvers.

\section{Numerical simulations for the case with uncertainties}

Figure 10. Comparison of the sets $Z_{1}$ of three models for $\psi=\pi / 2$

Consider now the case of lateral avoidance when some parameters of the dynamics are known with uncertainty. The numerical results presented here are obtained using finite difference scheme for two player HJB equations. We take here the reference example parameters and we suppose that the intruder velocity is known with some uncertainty $v \in\left[-v_{\max }, v_{\max }\right]$. For the lateral avoidance the dynamics of the system is defined in (15). To study the sensitivity of the set $W_{1}$ with respect to the range of the uncertainty we compare the results obtained with of different maximum amplitudes, $v_{\max }=0.1$ and $v_{\max }=0.5$.

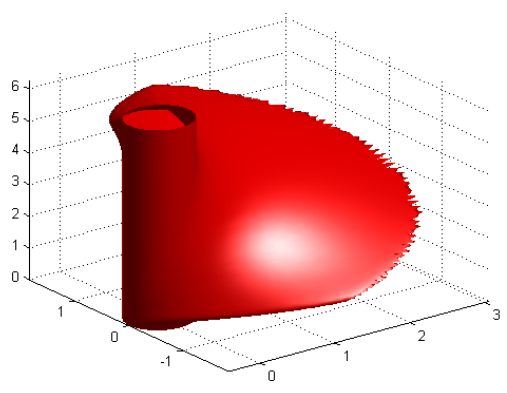

(a) $W_{1}$ in $3 \mathrm{D}, v_{\max }=0.1$

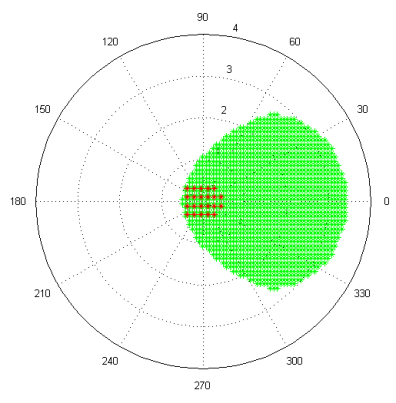

(b) Projection in $X Y$ plan: $v_{\max }=0.1$

Figure 11. The set $W_{1}$ for $v_{\max }=0.1$

The figure 11 shows the set $W_{1}$ when $v \in[-0.1,0.1]$.

Let now $v \in[-0.5,0.5]$. The figure 13 on the following page shows the comparison of the projections of the set $W_{1}$ for different values of the intruder speed.

\section{Acknowledgments}

This works was partially supported by the "DGA - Research department in the French Defence Ministry", under the Grant ENSTA-DGA number 0660037, and partially supported by the EU under the 7th Framework 


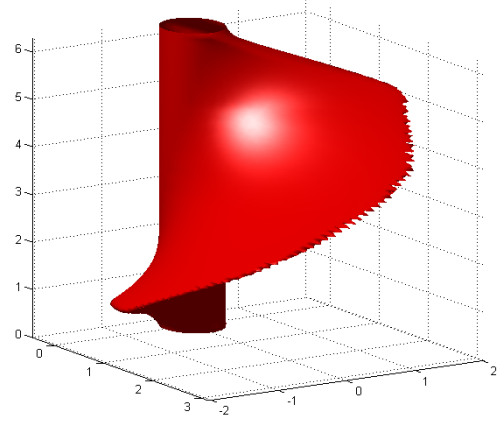

(a) $W_{1}$ in $3 \mathrm{D}, v_{\max }=0.5$

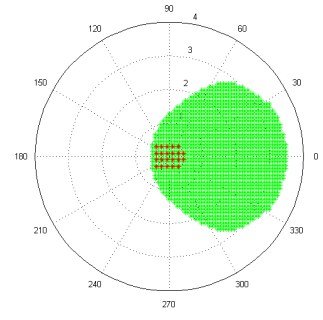

(b) Projection in $X Y$ plan: $v_{\max }=0.5$

Figure 12. The set $W_{1}$ for $v_{\max }=0.5$

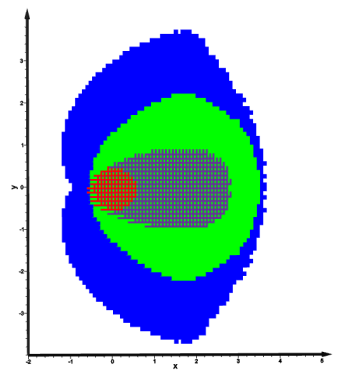

Figure 13. Comparison of three projections, when $v_{\max }=0.5$

Programme Marie Curie Initial Training Network FP7-PEOPLE-2010-ITN, SADCO project, GA number 264735-SADCO.

\section{References}

${ }^{1}$ http://www.air4all.net/.

${ }^{2}$ http://www.midcas.org/.

${ }^{3}$ EUROCONTROL Specifications for the use of military unmanned aerial vehicles as operational air traffic outside segregated airspace. Available at http://www.eurocontrol.int, April 2006. The referred version is Edition 0.9.

${ }^{4}$ Albert Altarovici, Olivier Bokanowski, and Hasnaa Zidani. A general Hamilton-Jacobi framework for nonlinear stateconstrained control problems. 2011. Submitted.

${ }^{5}$ O. Alvarez, E. Carlini, R. Monneau, and E. Rouy. Convergence for a first order scheme for a non-local eikonal equation. Appl. Numer. Math., 56(9):1136-1146, 2006.

${ }^{6}$ J.-P. Aubin. Viability Theory. Birkhäuser, Boston, 1991.

${ }^{7}$ J.-P. Aubin and A. Cellina. Differential inclusions, volume 264 of Comprehensive studies in mathematics. Springer, Berlin, Heidelberg, New York, Tokyo, 1984.

${ }^{8} \mathrm{M}$. Bardi and I. Cappuzzo-Dolcetta. Optimal control and viscosity solutions of Hamilton-Jacobi-Bellman equations, with appendices by M. Falcone and P. Soravia. Systems and Control: Foundations and Applications. Birkhäuser, Boston, 1997.

${ }^{9}$ O. Bokanowski and H. Desilles, A. Zidani. Hamilton-Jacobi approach for planning motion and reachability analysis. In Proceedings of ValueTools'2011 conference, to appear.

${ }^{10}$ O. Bokanowski, N. Forcadel, and H. Zidani. Deterministic state constrained optimal control problems without controllability assumptions. To appear in ESAIM: COCV, page DOI: 10.1051/cocv/2010030, 2010.

${ }^{11} \mathrm{O}$. Bokanowski, N. Forcadel, and H. Zidani. Reachability and minimal times for state constrained nonlinear problems without any controllability assumption. SIAM J. Control Optim., 48(7):4292-4316, 2010. 
${ }^{12}$ O. Bokanowski, S. Martin, R. Munos, and H. Zidani. An anti-diffusive scheme for viability problems. Applied Numerical Mathematics, 56(Issue 9, in Numerical Methods for Viscosity Solutions and Applications):1135-1254, 2006.

${ }^{13} \mathrm{O}$. Bokanowski and H. Zidani. Anti-diffusive schemes for linear advection and application to Hamilton-Jacobi-Bellman equations. J. Sci. Computing, 30(1):1-33, 2007.

${ }^{14} \mathrm{P}$. Cardaliaguet. A differential game with two players and one target. SIAM Journal on Control and Optimization, 34(4):1441-1460, 1996.

${ }^{15} \mathrm{P}$. Cardaliaguet, M. Quincampoix, and P. Saint-Pierre. Optimal times for constrained nonlinear control problems without local controllability. Appl. Math. Optim, 36:21-42, 1997.

${ }^{16}$ P. Cardaliaguet, M. Quincampoix, and P. Saint-Pierre. Pursuit differential games with state constraints. SIAM J. Control Optim., 39(5):1615-1632 (electronic), 2000.

${ }^{17}$ M.G. Crandall and P.-L. Lions. Two approximations of solutions of Hamilton Jacobi equations. Math. Comp., 43:1-19, 1984.

${ }^{18}$ E. Cristiani and M. Falcone. Fast semi-Lagrangian schemes for the Eikonal equation and applications. SIAM J. Numer. Anal., 45(5):1979-2011 (electronic), 2007.

${ }^{19}$ E. Crück and J. Lygeros. Sense and avoid system for a male uav. In AIAA GNS, Hilton Head, South Carolina, USA, August 2007.

${ }^{20}$ Jerry Ding, Eugene Li, Haomiao Huang, and Claire J. Tomlin. Reachability-based synthesis of feedback policies for motion planning under bounded disturbances. In ICRA, pages 2160-2165. IEEE, 2011.

${ }^{21}$ R. J. Elliott and N. J. Kalton. The existence of value in differential games. American Mathematical Society, Providence, R.I., 1972. Memoirs of the American Mathematical Society, No. 126.

${ }^{22} \mathrm{M}$. Falcone and R. Ferretti. Semi-lagrangian schemes for Hamilton-Jacobi equations, discrete representation formulae and godunov methods. J. Computational Physics, 175:559-575, 2002.

${ }^{23} \mathrm{M}$. Falcone, T. Giorgi, and P. Loreti. Level sets of viscosity solutions : some applications to fronts and rendez-vous problems. SIAM J. Applied Mathematics, 54(5):1335-1354, 1994

${ }^{24}$ L. Grüne. Adaptive grid generation for evolutive Hamilton-Jacobi-Bellman equations. In Numerical methods for viscosity solutions and applications (Heraklion, 1999), volume 59 of Ser. Adv. Math. Appl. Sci., pages 153-172. World Sci. Publ., River Edge, NJ, 2001.

${ }^{25}$ Gabriel M. Hoffmann and Claire J. Tomlin. Decentralized cooperative collision avoidance for acceleration constrained vehicles. In Proceedings of the 47th IEEE Conference on Decision and Control, 2008.

${ }^{26}$ David G. Hull. Fundamentals of Airplane Flight Mechanics. Springer, 2007.

${ }^{27}$ ASTM International. Standard Specification for Design and Performance of an Airborne Sense-and-Avoid System. Available for purchase at http://www.astm.org, July 2005. The referred version is designated F $2411-04^{\epsilon}$.

${ }^{28} \mathrm{H}$. Ishii. Uniqueness of unbounded viscosity solution of Hamilton-Jacobi equations. Indiana Univ. Math. Journal, 33(5):721-748, 1984.

${ }^{29}$ Michael P. Vitusy Jeremy H. Gillulay, Haomiao Huangy and Claire J. Tomlin. Design of guaranteed safe maneuvers using reachable sets: Autonomous quadrotor aerobatics in theory and practice. In 2010 IEEE International Conference on Robotics and Automation Anchorage Convention District, 2010.

${ }^{30}$ G.-S. Jiang and D. Peng. Weighted ENO schemes for Hamilton-Jacobi equations. SIAM J. Sci. Comput., 21(6):2126-2143 (electronic), 2000.

${ }^{31}$ Andrew Lacher, Andrew Zeitlin, David Maroney, Kelly Markin, Duane Ludwig, and Joe Boyd. Airspace integration alternatives for unmanned aircraft. In AUVSI's Unmanned Systems Asia-Pacific, Singapor, 2010.

${ }^{32}$ J. Lygeros. On reachability and minimum cost optimal control. Automatica, 40:917-927, 2004.

${ }^{33}$ J. Lygeros, C. Tomlin, and S. Sastry. Controllers for reachability specifications for hybrid systems. Automatica, 35:349$370,1999$.

${ }^{34}$ K. Margellos and J. Lygeros. Hamilton-Jacobi formulation for Reach-Avoid Differential Games. Technical report, November 2009 .

${ }^{35}$ K. Margellos and J. Lygeros. Hamilton-Jacobi formulation for Reach-Avoid Problems with an application to Air Traffic Management. In American Control Conference, Baltimore, MD, USA, June 2010.

${ }^{36}$ I. Mitchell, A. Bayen, and C. Tomlin. A time-dependent Hamiliton-Jacobi formulation of reachable sets for continuous dynamic games. IEEE Transactions on automatic control, 50(7):947-957, 2005.

${ }^{37} \mathrm{~S}$. Osher. A level set formulation for the solution of the Hamilton-Jacobi equations. SIAM J. Math. Anal., 24(5):11451152, 1993.

${ }^{38}$ S. Osher and C-W. Shu. High essentially nonoscillatory schemes for Hamilton-Jacobi equations. SIAM J. Numer. Anal., 28(4):907-922, 1991.

${ }^{39}$ P. Saint-Pierre. Approximation of the viability kernel. Applied Mathematics and Optimisation, 29:187-209, 1994.

${ }^{40}$ P. Saint-Pierre. Approximation of the viability kernel. Appl. Math. Optim, 29:187-2009, 1994. 